\title{
Real Time Simulation of Automatic Generation Control For Interconnected Power System
}

\author{
Naimul Hasan, Ibraheem, Shuaib Farooq \\ Department of Electrical Engineering \\ Jamia Millia Islamia, New Delhi-110025, India \\ naimul_hasan@rediffmail.com
}

\begin{abstract}
This paper presents real time simulation to analyze the behavior of discrete controller for interconnected power system. A two-area interconnected power system consisting of non-identical power plants with EHVAC transmission link as interconnection is considered for investigations. The hydro and nuclear power plant comprises of area-1 and area-2 respectively. In this paper new control strategies for digital controller is developed depending on the sampling time to test the controller in the non real time simulation environment and transform the controller into a real-time prototype controller, which is used for testing the algorithm in real working conditions. The real time simulation provides a quick solution for prototyping new functions in different types of industrial processes and devices controlled with a complex distributed control system. The real time simulation uses the discrete model of the AGC. Hence discrete controller for the AGC is derived from the continuous controller by discritization of the continuous controller using Tustin transformation, or trapezoid approximation method and the closed loop stability is checked by taking the bode of the closed loop of AGC model for different sampling time periods ,the dynamic response plot for $1 \%$ load disturbance in area- 1 . The discrete controller is prototyped and the real time simulation is performed to observe the results, the dynamic response plots are obtained for $1 \%$ load disturbance in area- 1 of the real time model.
\end{abstract}

Keywords: AGC, control prototyping, hydro-nuclear interconnected power system, real-time simulation.

\section{Introduction}

In real situations, the power systems consist of conventional forms of electrical power generations like, thermal, hydro, and nuclear as a major share of electrical power. The configuration of today's integrated power system becomes more complex due to these power plants with widely varying dynamic characteristics. Nuclear units owing to their high efficiency are usually kept at base load close to their maximum output with no participation in system automatic generation control (AGC) [1-3]. Gas power generation is ideal for meeting varying load demand. However, such plants do not play very significant role in AGC of a large power system, since these plants form a very small percentage of total system generation. Gas plants are used to meet peak demands only. Thus the natural choice for AGC falls on either thermal or hydro units. But with integration of nuclear power plants in the power system, it is also required to study the behaviour of AGC for the interconnected power system considering nuclear power plant. A literature survey shows that most of the earlier works in the area of AGC pertain to interconnected thermal systems and relatively lesser attention has been devoted to the AGC of interconnected hydro nuclear system [4-5]. The generating characteristics of low-head hydro units such as used in run-of-river plants have excellent response capabilities.

Received: July $3^{\text {rd }}, 2011$. Accepted: December $27^{\text {th }}, 2011$ 
Many can be cycled over their entire operating range in under a minute. High-head units must have their response rates somewhat curtailed to prevent water-hammer damage in long penstocks. Even with such a limitation, the units can respond with very large excursions, if desired. However, hydro plant loading maneuvers may require careful coordination with other hydro units upstream or downstream on the same river system and the most of the nuclear units have either boiling-water (BWR) or pressurized-water (PWR) steam generators. Most are not currently controlled by AGC, but there are exceptions. BWR units operate under AGC typically can respond at $3 \%$ per minute for 10 minutes or so within their regulating range. To move outside the range requires making changes manually in the control-rod pattern, a more lengthy process. Power control in PWR units is accomplished by adjusting control rods in the reactor core, and for larger excursions at slower rates, by changing the concentration of boric acid in the primary loop. These units are capable of making $20 \%$ excursions at rates of nearly $3 \%$ per minute.

The objectives traditionally defined for AGC appear to be vague and incomplete. Only comparison of attributes of AGC strategies from different aspects and for each attribute, the preferred strategy is indicated. The concepts developed for the single control area case are then extended to that of an interconnection comprising several control areas. Supplementary controllers are designed to regulate the area control errors to zero effectively. Several modern design techniques have been used to optimize the parameters of the supplementary controllers and these techniques are usually analyzed in off-line simulation using variable step solvers. As the controllers are usually implemented in discrete mode therefore the discrete time analysis of the control strategies is to be done before implementing in field. Rapid control prototyping (RCP) is a control design method where testing of a new controller is done first in simulation environment. The simulation models are then converted with aid of automatic code generation to a prototype controller that can be used in field testing. With this approach, the time needed for developing new functions is reduced significantly because the manual computer code implementation phase is left out of the design and testing process. Consequently, it is possible to evaluate different control solutions without additional delay.

\section{Power System Model under Investigation}

Investigations have been carried out on an interconnected Hydro-Nuclear system as shown in figure1, neglecting the generation rate constraints.

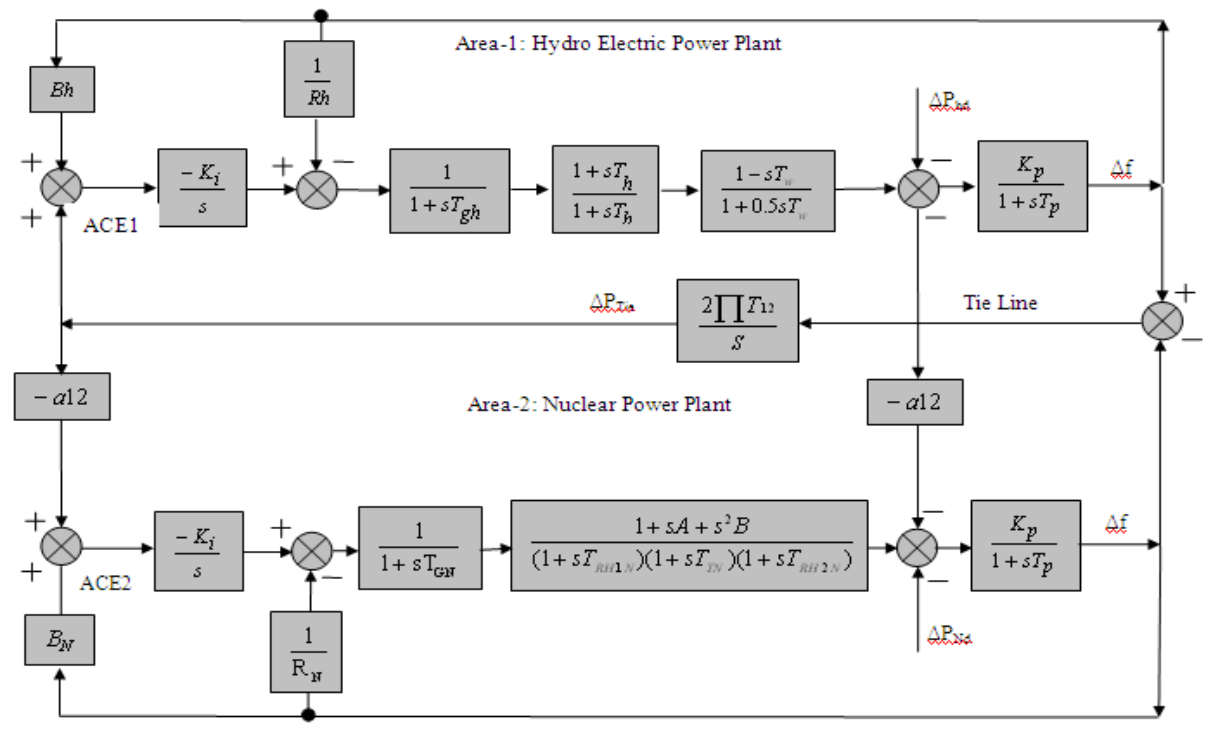

Figure 1. Transfer function model of an interconnected two-area Hydro- Nuclear system. 


\section{System Model}

Mathematical model is developed and step load perturbation of $1 \%$ of nominal loading has been considered in area-1. to study the dynamic behavior of the system [6-9]. Below is the mathematical of each of the components needed to build the power system model.

\section{A. Generator}

The Generator dynamics is modeled by swing equation and is given in equation. 1

$$
\frac{2 H}{\omega_{s}} \frac{d^{2} \Delta \delta}{d t^{2}}=\Delta P_{m}-\Delta P_{e}
$$

For small perturbation the above relation can be represented by a block diagram shown in figure 2

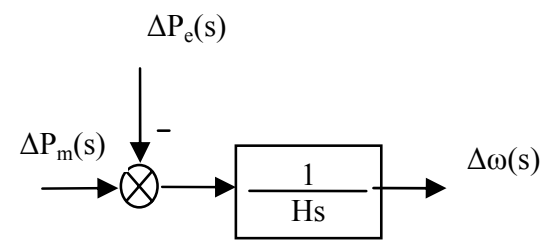

Figure 2. Transfer function diagram of generator

Similarly the composite load is considered and the corresponding transfer function for the load model is given as-

$$
\Delta \mathrm{P}_{\mathrm{e}}=\Delta \mathrm{P}_{\mathrm{L}}+\mathrm{D} \Delta \mathrm{W}
$$

Where $\Delta \mathrm{PL}$ is the non frequency-sensitive load change and $\mathrm{D} \Delta \omega$ is the frequency sensitive load change. $\mathrm{D}$ is expressed as percentage change in load divided by the percentage change in frequency.

Therefore the combined transfer function for the generator load model is shown in figure 3.

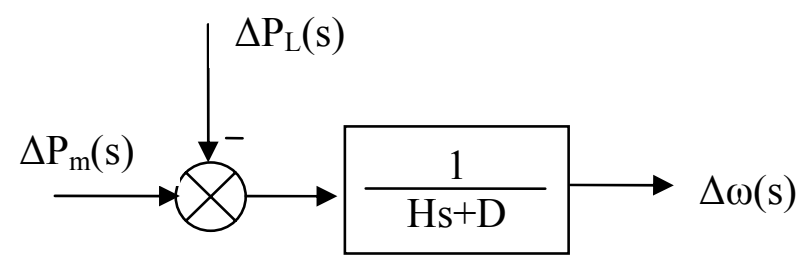

Figure 3. Combined transfer function diagram of generator and load

\section{B. Tie Line}

The Tie line power is represented in equation 3 and the corresponding block diagram developed from equation $3,4,5$ is given in figure 4 .

$$
P_{\text {tie }, 1}=\frac{\left|V_{1}\right|\left|V_{2}\right|}{X_{12}} \operatorname{Sin}\left(\delta_{1}^{0}-\delta_{2}^{0}\right)
$$


Where, $\delta_{1}^{0}, \delta_{2}^{0}=$ Power angles of equivalent machines of the two areas.

For incremental changes in $\delta_{1}$ and $\delta_{2}$ the incremental tie line power can be expressed as.

$$
\Delta P_{\text {tie }, 1}(p u)=T_{12}\left(\Delta \delta_{1}-\Delta \delta_{2}\right)
$$

Since incremental of power angles are integral of incremental frequencies, can be written as

$$
\Delta P_{\text {tie, } 1}(p u)=2 \pi T_{12}\left(\int \Delta f_{1} d t-\int \Delta f_{2} d t\right)
$$

Where $\Delta \mathrm{f}_{1}$ and $\Delta \mathrm{f}_{2}$ are incremental frequency changes of Area-1 and Area-2 respectively.

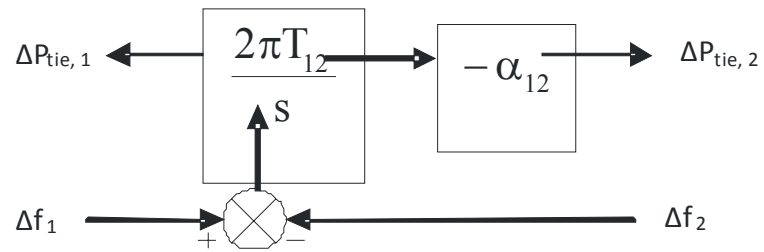

Figure 4. Isolated model of hydro power system for LFC

\section{Hydraulic Turbine Governor System}

The transfer function for the mechanical hydraulic governor is given by eq 6 and the turbine is given by eq 7 :

$$
\begin{aligned}
& T(H-\text { governor })=\left(\frac{K_{g h 2}}{1+s T_{g h 2}}\right)\left(\frac{1+s T_{h 2}}{1+s T_{h 4}}\right) \\
& T(H)=\frac{1-s T_{h 2}}{1+0.5 s T_{h 4}}
\end{aligned}
$$

Where, $\mathrm{K}$ and $\mathrm{T}$ are the gain and time constant of the hydraulic system (h, 2 and 4 are suffix for Hydro area and $\mathrm{g}$ for governor).

The combined model for isolated hydro power system is given in figure 5 .

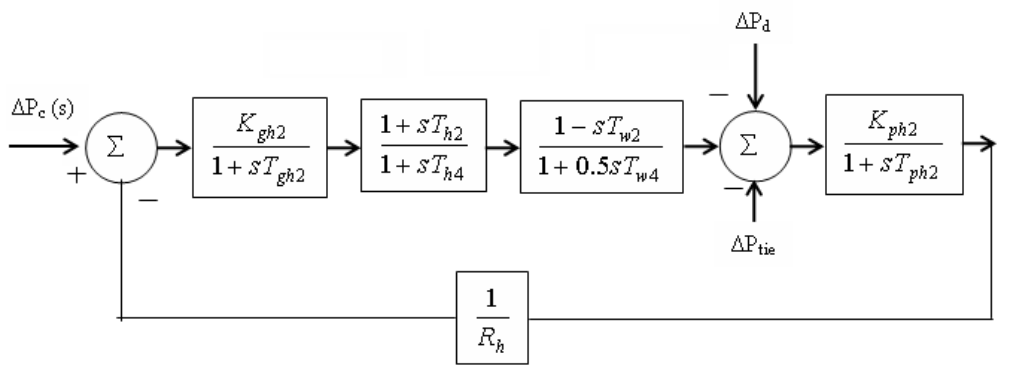

Figure 5. Isolated model of hydro power system for LFC

\section{Nuclear Turbine Governor System}

The Mathematical model considered for Nuclear unit with tandem-compound turbines, one HP section and two LP sections with HP reheater is shown in fig 6 [10-11]. 


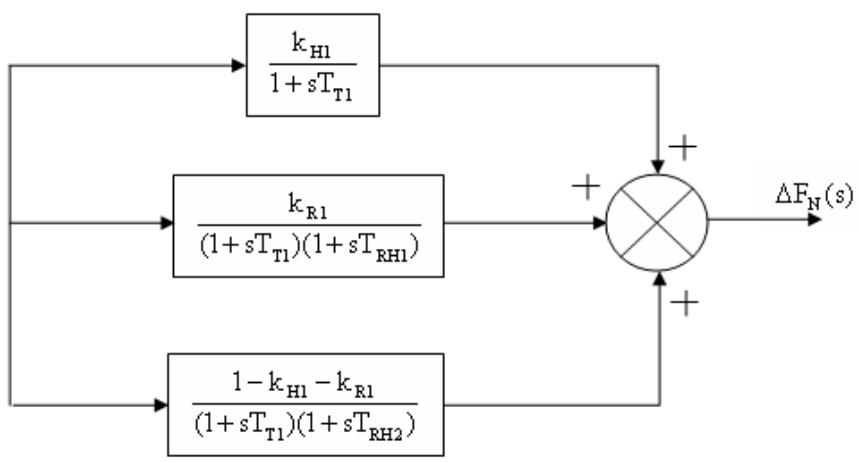

Figure 6. Isolated model of Nuclear Power System for LFC

\section{Controller Model and Tuning}

In this paper a two area interconnected power system with area 1 comprises of hydro power system and area 2 comprises of nuclear power system and conventional PI Controller is considered.

1. The output of conventional PI controller is given in equation 8 .

$$
U(t)=K_{p}\left(e(t)+1 / T_{i} \int e(t) d t\right)
$$

The control signal depends upon error signal e(t). In this case e $(t)$ is area control error (ACE). Where $\mathrm{Kp}$ is proportional gain, Ti is the integral times and Area Control Error (ACE) is expressed as linear combination of incremental frequency and tie line power. Thus ACE for control Area-1 and for control Area-2 are given as:-

$$
\begin{aligned}
& \operatorname{ACE}_{1}(\mathrm{~s})=\Delta \mathrm{P}_{\text {tie, }, 1}(\mathrm{~s})+\mathrm{B}_{1} \Delta \mathrm{f}_{1}(\mathrm{~s}) \\
& \mathrm{ACE}_{2}(\mathrm{~s})=\Delta \mathrm{P}_{\text {tie }, 2}(\mathrm{~s})+\mathrm{B}_{2} \Delta \mathrm{f}_{2}(\mathrm{~s})
\end{aligned}
$$

For tuning the gain of the controller, the investigated system is first considered without AGC controllers and then 1\% step load perturbation is given in both the areas and Integral Square Error criterion is used to obtain optimum gain parameters for Controllers. In the beginning the integral gains are assumed zero and optimum value of proportional gain $(\mathrm{Kp})$ is obtained. With this value of $\mathrm{Kp}$, integral gain is optimized using ISE criterion. The dynamic performance of the AGC system depends upon controllers gain setting hence optimized gains are calculated by the ISE techniques whose performance index is given as

$$
J=\int_{0}^{t}\left(\alpha \Delta f 1^{2}+\beta \Delta f 2^{2}+\Delta P_{t i e}{ }^{2}\right) d t
$$

A value of 0.65 is used for $\alpha$ and $\beta$. and after several iteration the optimized value of gain 0.03 is calculated.

\section{Digital Controller Model and Tuning}

Different techniques are used to convert continuous systems into discrete systems but the continuous system can only be approximated and the discrete system can never be exactly equivalent. Different methods can result different controller performances [12-14]. The method used in this paper is called the Tustin transformation or trapezoid approximation and equation is given as:- 


$$
S=\frac{2}{T}\left(\frac{Z-1}{z+1}\right)
$$

Where $\mathrm{T}$ is the sampling time that depends on the time constant of the system and the approximation taken here is represented by equation 13 .

$$
\frac{\sum T k+T h}{25}<T<\frac{25}{12} \frac{\sum T k+T h}{25}
$$

In this paper continuous time PI controller is discretized with various different sampling periods and has been observed that the stability of the equivalent discrete controller becomes smaller as the sampling period reduces and the response of the system is unbounded. Therefore sampling time and gain of the controller is tuned taken so that the design criteria of minimum settling time and the closed-loop system should be stable. The discritization is done with a sampling time of $0.1 \mathrm{sec}$ and behavior is compared and analyzed with the continuous time controller by plotting Bode plots as shown in figure 7.

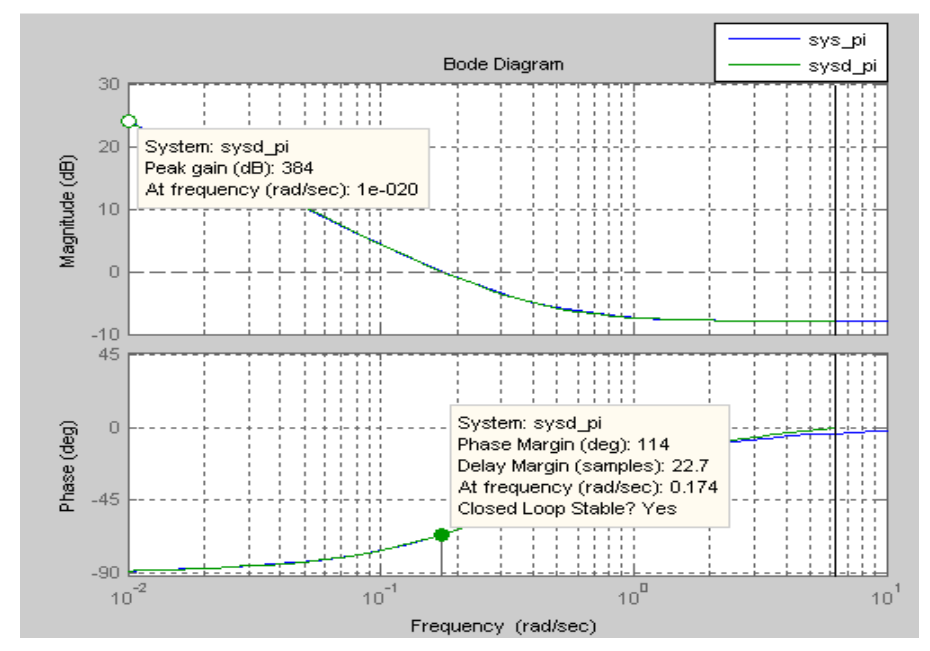

Figure 7. Bode of continuous and discrete PI controller

\section{Simulation Studies}

AGC controllers are designed to regulate the area control errors to zero effectively and the design techniques have been used to optimize the parameters of the supplementary controllers by analyzing the dynamic behavior in off-line simulation that may be time-consuming, numerically instable due to variable step solvers and lack the capability of interfacing with real devices. A real-time simulation is therefore gradually adopted to replace off-line simulation without the mentioned drawbacks. The significant requirement of the real-time simulation is to ensure that the calculation for a time-step is accomplished within the chosen step-size that is usually known as hard real time simulation.

In this paper the following simulation has been done and observations have been discussed and compared.

(i). Non-Real time Simulation of continuous time controller known as continuous time model and discrete time controller known as hybrid model of AGC for Hydro-Nuclear interconnected power system.

(ii). Real time Simulation of discrete time controller (Hybrid model) of AGC for HydroNuclear interconnected power system. 
For the real time implementation, the controller is discretized and coded to run as a real time task under control of the real time operating system. The block diagram of hybrid system employed in this paper is shown in figure 8 .

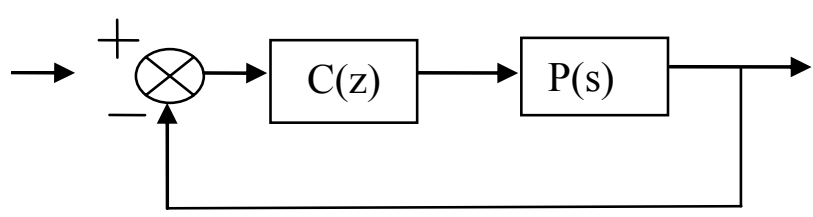

Figure 8. Discrete controller plant feedback configuration

\section{Hardware and Software Architecture For Real Time Simulation}

In this work single PC loaded with the Microsoft Windows, MATLAB with Real time Windows target and $\mathrm{C} / \mathrm{C}++$ Compiler is used for prototyping digital controller the setup configuration is shown in fig 9. The real time exchange of data between continuous plant model and the digital controller is via MATLAB real time windows kernel that will run the controller in hard real time.
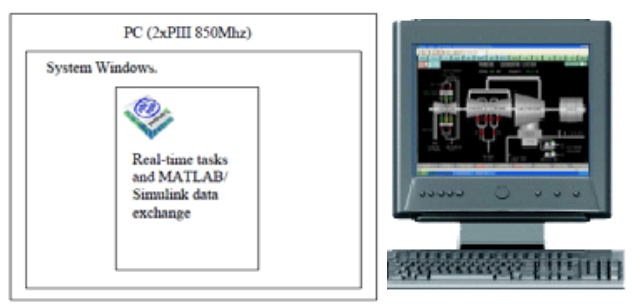

Figure 9. Standalone Simulation system

\section{Non Real Time Simulation}

In this study, the application of continuous time and discrete time PI controller to AGC in the power system with two areas having hydro-nuclear turbine tied together power line is investigated. The load perturbation or perturbations having amplitude of 0.01 p.u.MW to area 1 is applied and the frequency oscillations and tie-line power flows are investigated. The simulations are implemented using MATLAB / SIMULINK and the comparison due to load perturbation is observed. Simulation of below systems are done and dynamic behavior is observed and compared.

(i). Continuous time PI controller with continuous time power system model (Continuous system).

(ii). Discrete time PI controller with continuous time power system (Hybrid system).

AGC model with continuous time controller as shown in fig 10.0 and the same model with equivalent digital PI controller as shown in fig 11.0 is simulated with load perturbation having amplitude of 0.01 p.u.MW to area 1, the frequency oscillations and tie-line power flows are observed and the comparison are made.

The results obtained from the optimum value of continuous time controller gains and the discrete time controller are shown in fig 12.0.Examining the responses it is seen that continuous time PI controller take more settling time and max peak deviation than the discrete time PI controller when perturbation of $1 \%$ occurs in the hydro area. 


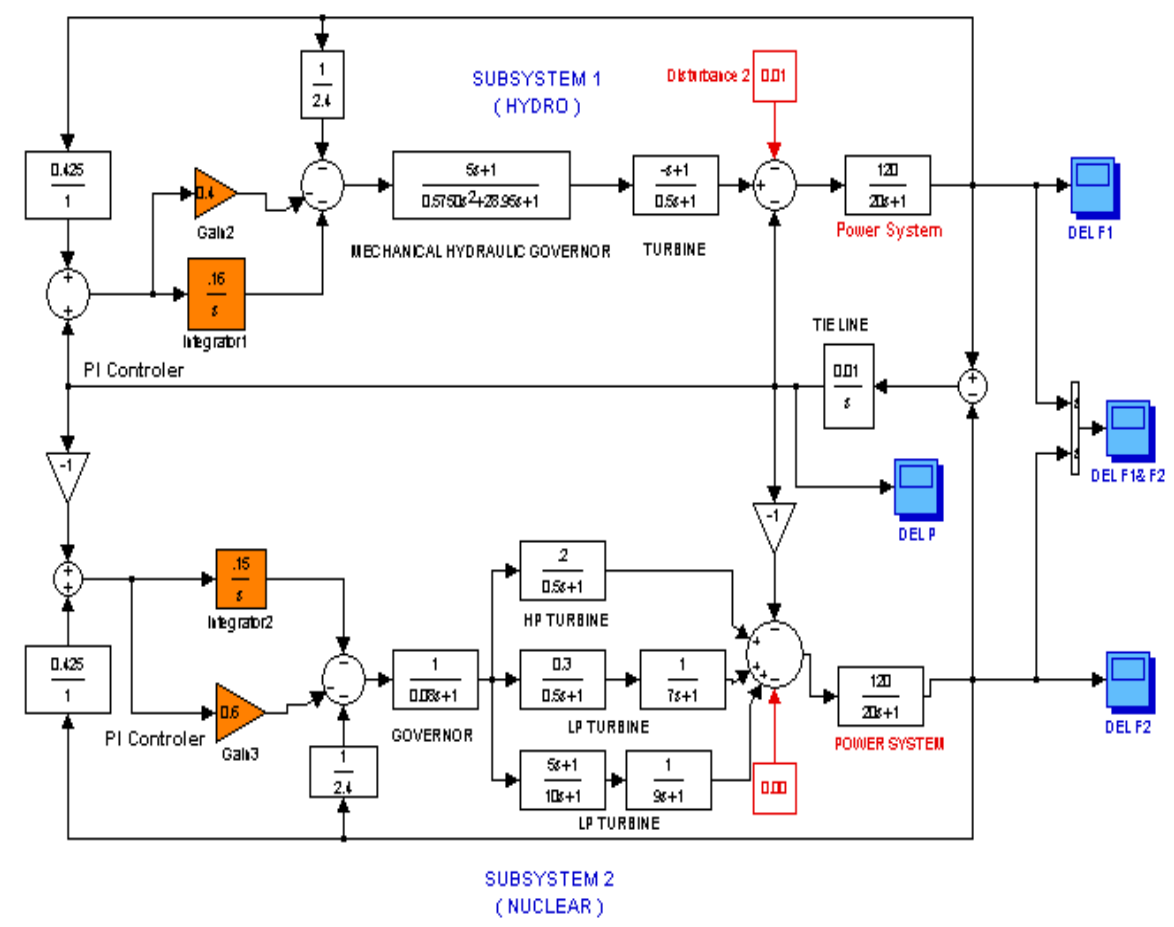

Figure 10. AGC Model with continuous PI controller

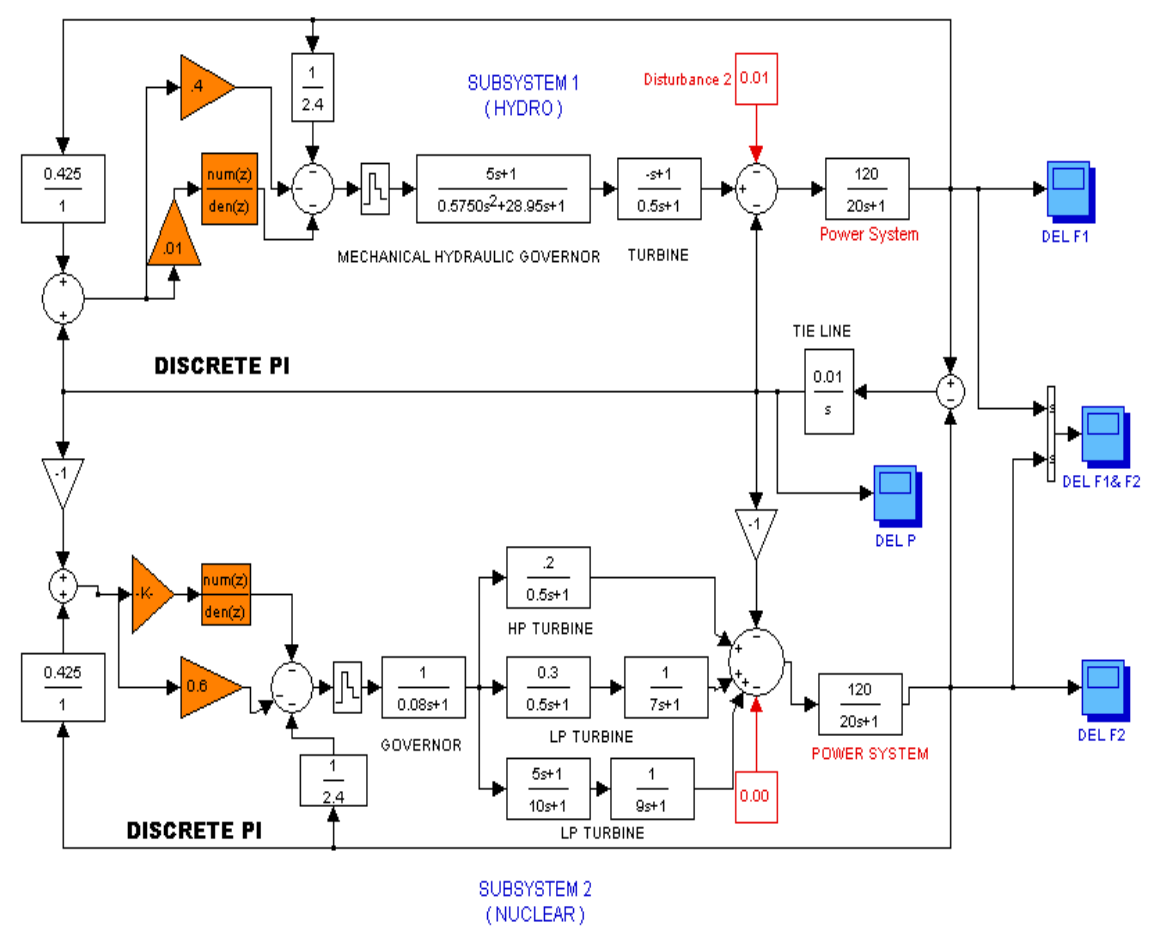

Figure 11. AGC Model with discrete time PI controller 


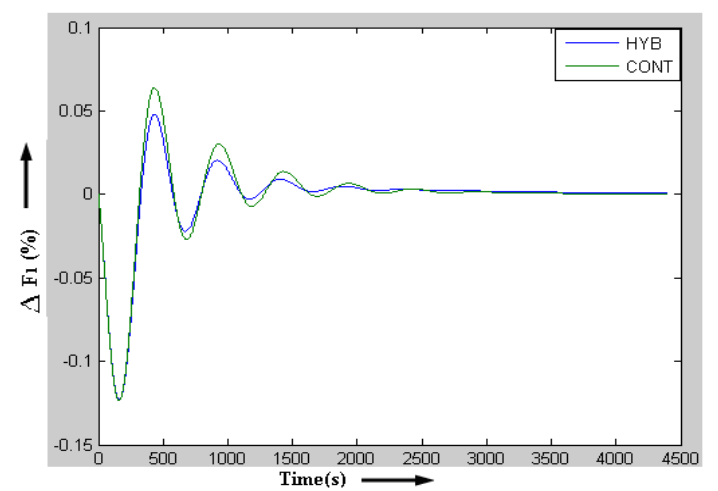

Figure 12. Time response of $\Delta \mathrm{F} 1$ for $1 \%$ load disturbance in area-1

As shown in figure 13.0 for the deviation in tie line power the continuous time PI controller take more settling time than the discrete time PI controller.

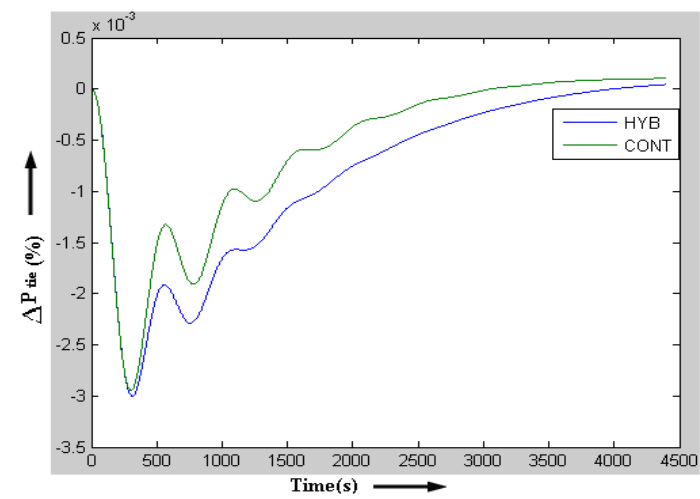

Figure 13. Time response of $\Delta$ Ptie for $1 \%$ load disturbance in area-1

Examining the responses it is clear that the discrete controller improves effectively the damping of the Oscillations after the load deviation in one of the areas in the interconnected power system as compared to continuous controller and also has been observed, the first peak of the hybrid model and the continuous model are same where as the oscillations and settling time are less for the hybrid model as compared to continuous model.

\section{Real Time Simulation}

Hard Real time simulation is performed to implement the discrete controller on microprocessor [15-20]. The hardware and software architecture as discussed in section VII is used for the real time simulation of the discrete controller. MATLAB /SIMULINK/ REAL TIME WORKSHOP/WATCOM C++ Compiler are used to generate the corresponding $\mathrm{C}$ code, compiling and linking to the target machine. Comparison is studied between the real time simulation and non-real time simulation of PI controller for hydro-nuclear interconnected power system as shown in fig 14.0 , the results obtained are shown in fig 15.0 and fig 16.0 by examining the response it has been observed that the first negative peak, oscillations and settling time of the real time model is better than the non-real time model however response of the real time model depends on the fixed time step which is taken as $0.01 \mathrm{sec}$ in this paper. 


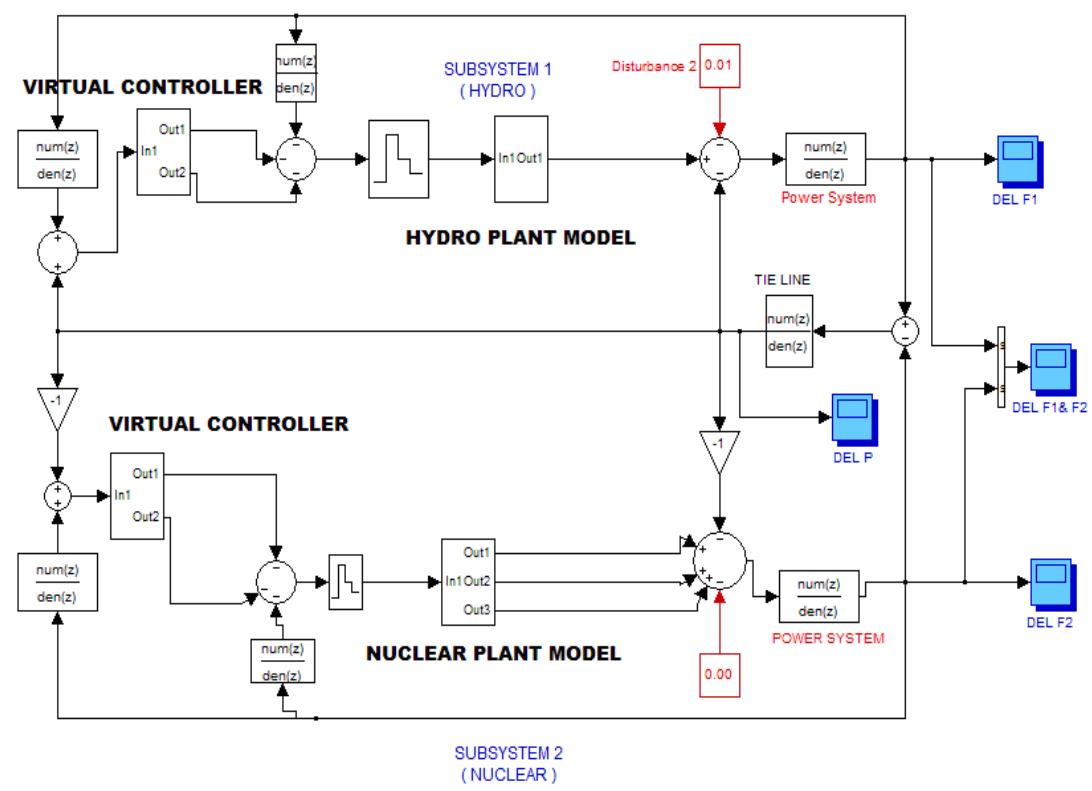

Figure 14. Real-time simulation of two area hydro-nuclear power system with discrete PI controller

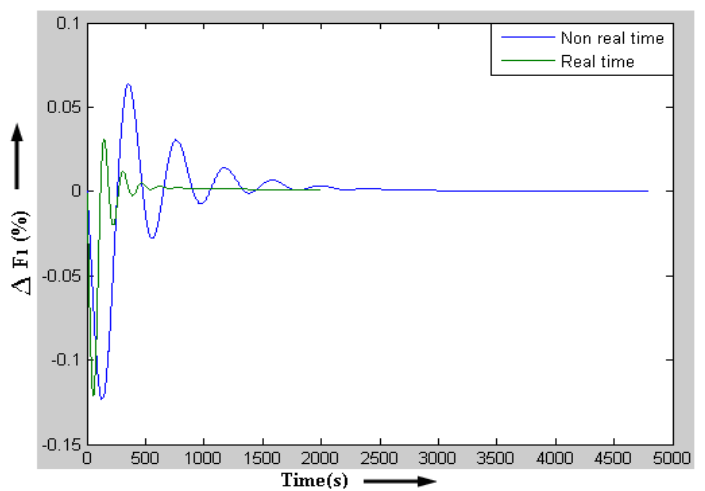

Figure 15. Time response of $\Delta \mathrm{F} 1$ for $1 \%$ load disturbance in area-1

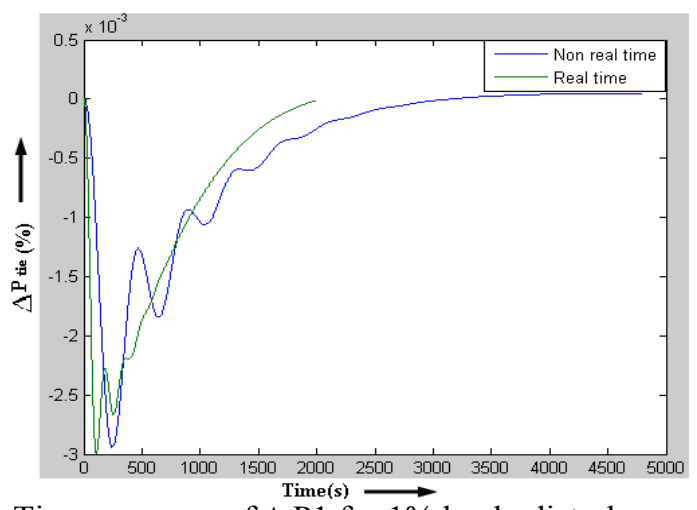

Figure16. Time response of $\Delta \mathrm{P} 1$ for $1 \%$ load disturbance in area-1 
The real time simulation of controllers have the following advantages over the non-real time simulation. Prototyping of the discrete controller can easily be developed on any PC or any target microprocessor so that behavior of the controller can be verified along with the mathematical model and the hardware. The on-line tuning of the gain can easily be developed. Then the discrete controller of power system (hybrid system) is prototyped on Intel Pentium 1.70 Ghz with 1GB RAM using Simulink-Real time windows target and Watcom C compiler and comparison is observed for the non real-time simulation and real-time simulation.

\section{Conclusion}

The real time simulation uses the discrete model of the AGC. Hence discrete controller for the AGC is derived from the continuous controller by discritization of the continuous controller using Tustin transformation, or trapezoid approximation method and the closed loop stability is checked by taking the bode of the closed loop of AGC model for different sampling time periods ,the dynamic response plot for $1 \%$ load disturbance in area-1 is quite appreciable for the hybrid model (continuous model with discrete controller) as compared to continuous model for the frequency deviation in area 1 and in tie line power. When discrete controller is prototyped and the real time simulation is performed to observe the results, the dynamic response plot for $1 \%$ load disturbance in area- 1 of the real time model is quite appreciable as compared to non real time model. The peak magnitude, oscillations and settling time are quite low as compared to non real time model. Real time simulation is good technique of prototyping the controllers for various applications in the lab.

\section{References}

[1] C. Concordia and L. K. Kirchmayer, "Tie-line power \& frequency control of electric power system: Part II,” AISE Trans, III-A, vol. 73, pp. 133-146, Apr. 1954.

[2] L. K. Kirchmayer, Economic Control of Interconnected Systems. New York: Wiley, 1959.

[3] Ibraheem, P. Kumar and D.P. Kothari, "Recent philosophies of automatic generation control strategies in power systems," IEEE Transaction on Power Apparatus System 20 (1) (2005), pp. 346-357.

[4] "IEEE Transaction on Power App. System.," IEEE Committee Rep., vol. PAS-86,pp. 384-395, 1966.

[5] M. L. Kothari, B. L. Kaul, and J. Nanda, "Automatic generation control of hydrothermal system,” J. Inst. Eng. India, pt. EL2, vol. 61, pp. 85-91, Oct. 1980.

[6] F. R. Schleif and A. B. Wilbor, "The co-ordination of hydraulic turbine governors for power system operation," IEEE Transaction on Power Apparatus System vol. PAS-85, no. 7, pp. 750-758, Jul. 1966.

[7] D. G. Ramey and J. W. Skooglund, "Detailed hydro governor representation for system stability studies," IEEE Transaction on Power Apparatus System vol. PAS-89, no. 1, pp. 106-112, Jan. 1970

[8] K.P. Singh Parmar, S. Majhi and D.P. Kothari, Automatic Generation Control of an Interconnected Hydrothermal Power System, IEEE Conf. proceedings, INDICON 2010, Kolkata, India.

[9] D. G. Ramey and J. W. Skooglund, "Detailed hydro governor representation for system stability studies," IEEE Transaction on Power Apparatus System vol. PAS-89, no. 1, pp. 106-112, Jan. 1970.

[10] T. Ichikawa, "Dynamics of Nuclear Power Plant in Electric Power System (Part 1)-BWR plant," CRIEPI Report No. 175079, July 1976

[11] T. Ichikawa, "Nuclear Power Plant Dynamics in Electric Power System (part 2)-BWR Plant Dynamics Simulation Model,” CRIEPI Report No. 176072, July 1977. 
[12] M. L. Kothari, J. Nanda, and L. Hari, "Selection of sampling period for automatic generation control," Int. J. Elect. Mach. Power Syst., vol. 25, no. 10, pp. 1063-1077, Dec. 1997.

[13] O. I. Elgerd and C. E. Fosha, "Optimum megawatt-frequency control of multi-area electric energy systems," IEEE Transaction on Power Apparatus. System, vol. PAS-89, no. 4, pp. 556-563, Apr. 1970.

[14] L. Hari, M. L. Kothari, and J. Nanda, "Optimum selection of speed regularization parameter for automatic generation control in discrete mode considering generation rates constraint," Proc. Inst. Elect. Eng., vol. 138, no. 5, pp. 401-406, Sep. 1991.

[15] C. Dufour and J. Belanger, "Real-time PC-based simulator of electric systems and drives," in Proc. Int. Conf. on Parallel Computing Electrical Engineering, Vol. 1, Sep. 2004, pp. 105-113.

[16] Grega W., Kołek K.: Simulation and Real-time Control: from Simulink to Industrial Applications, Proceedings of 11th IEEE International Conference on Computer Aided Control System Design, Glasgow, 2002, pp. 104109.

[17] Camn W. Taylor R. Lee Chap "Real-time power system simulation for automatic generation control", IEEE Transactions on Power Apparatus and Systems, Vol. PAS-95, no. 1, January/February 1976.

[18] A. Gambier "Real-time control systems: Tutorial" 2004 5th Asian Control Conference.

[19] Aslaug Haraldsdottir "Simulation of a continuous system controlled by a digital controller," Technical report no. UM-MEAM 532.

[20] Peter H. Meckl, George T.-C. Chiu, Patricia Davies "Implementing digital controller: A case study", Proceedings of the American Control Conference San Diego, California June 1999

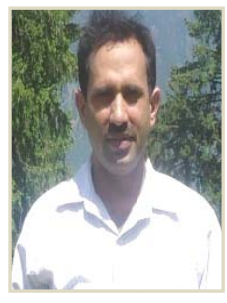

Naimul Hasan did his Ph.D. degree in Electrical Engineering from Department of Electrical Engineering, Jamia Millia Islamia, New Delhi, India. Presently he is an Asstt. Professor in the same Department. He has published a number of papers in International/National Journals and Conferences. His research area includes power system operation and control, energy management.

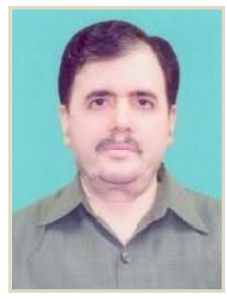

Ibraheem is presently Professor in the Department of Electrical Engineering, Jamia Millia Islamia (Central University), New Delhi, India. He has published a number of research papers in International/National Journals. His current activities include teaching and research in the areas of power system control, optimal control theory, suboptimal control of power systems, applications of soft computing techniques in power systems, and HVDC transmission systems. Dr. Ibraheem received a Gold Medal from the ministry of Power and Energy (India) in 1998 for one of research articles.

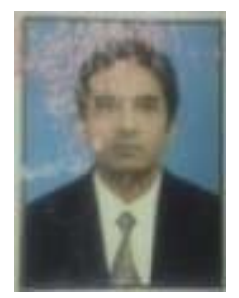

Suhaib Farooq is a research Scholar in the Department of Electrical Engineering, Jamia Millia Islamia (Central University), New Delhi, India. His major field of interest includes real time operation of power system and soft computing techniques. 